\title{
Treatment adherence and other patient-reported outcomes as cost determinants in multiple sclerosis: a review of the literature
}

This article was published in the following Dove Press journal:

Patient Preference and Adherence

4 December 2014

Number of times this article has been viewed

\author{
Luis Lizán' \\ Marta Comellas' \\ Silvia Paz' \\ José Luis Poveda \\ Dennis M Meletiche ${ }^{3}$ \\ Carlos Polanco ${ }^{4}$ \\ 'Outcomes' I 0, Castellón, Spain; \\ ${ }^{2}$ Hospital Universitario La Fe, Valencia, \\ Spain; ${ }^{3}$ EMD Serono Inc., Rockland, \\ MA, USA; ${ }^{4}$ Merck S.L., Madrid, Spain
}

Correspondence: Carlos Polanco Corporate Affairs, Merck SL, María de Molina, 40, 28006 Madrid, Spain

Tel +34 917453 I58

Fax +34917454 444

Email carlos.polanco@merckgroup.com
Background: Treatment adherence is one of the key factors for achieving optimal clinical outcomes. In order to assess costs related to adherence to, and persistence and compliance with, disease-modifying therapies (DMTs) in patients with multiple sclerosis (MS), a narrative review of the literature was performed. Satisfaction with and preference for DMTs and their delivery devices were also assessed, as both can have an influence on patients' adherence and persistence.

Methods: Electronic databases (MEDLINE, PubMed, Google Scholar, congress proceedings) were searched to identify publications analyzing MS costs related to adherence, persistence, satisfaction, and preferences for MS treatments. Bibliographic references were hand searched. English or Spanish studies published between January 2007 and January 2013 were selected.

Results: A total of 398 titles were identified, of which 12 met the inclusion criteria. Six studies evaluated the impact of adherence, persistence, and compliance on treatment costs; four publications analyzed satisfaction with DMTs; and two assessed treatment preferences based on attributes of the delivery device. Increased adherence and persistence were associated with better clinical outcomes, leading to lower relapse risk (odds ratio [OR]: 0.71; 95\% confidence interval [CI]: 0.59-0.85) and a decrease in health care resource use, such as MS-related hospitalizations (OR: 0.63; 95\% CI: 0.47-0.83) and emergency department visits (OR: 0.80; 95\% CI: $0.60-1.07)$. This reduction in resource use led to a patient/year total cost reduction (excluding DMT costs) of up to $22 \%$.

Conclusion: This review highlights the importance of ensuring adequate adherence in MS patients through treatments and devices better tailored to patients' needs that could enhance clinical outcomes and reduce MS costs. Understanding the factors underlying satisfaction and compliance with treatment and patients' preference for certain therapies could help in the development of strategies that can improve adherence.

Keywords: multiple sclerosis, adherence, satisfaction, delivery devices, costs

\section{Introduction}

Multiple sclerosis (MS) is an inflammatory, neurodegenerative disease of the central nervous system that results in demyelination and transaction of axons in the brain, spinal cord, and optic nerves. ${ }^{1}$ The clinical disease course is variable, usually starting with reversible episodes of neurological disability in the third or fourth decade of life, then transforming into a disease marked by continuous and irreversible neurological decline by the sixth or seventh decade. ${ }^{1}$ This clinical course may be considered the expression of two phenomena: relapses of acute neurological symptoms, which end in partial or complete remission; and progression, which refers to the steady and irreversible worsening of signs and symptoms. ${ }^{2,3}$ 
MS affects about 2 million-2.5 million people worldwide. ${ }^{4}$ In Europe, the prevalence tends to be higher in the United Kingdom and in Nordic countries, ranging from $96 / 100,000$ to more than $200 / 100,000$, compared with the situation in Central European countries, where prevalence ranges from $62 / 100,000$ to $128 / 100,000 .^{5}$ According to a recent analysis of MS incidence trends in the European Economic Area, the incidence of MS ranges from 1.12-6.96 per 100,000 persons, is higher in females, and triples with increasing latitude. ${ }^{6}$

Due to the early age of disease onset and its progressive disabling course, the economic burden of MS is considerable. Moreover, taking into account that MS affects people in the most productive stage of their lives, affecting patients' and caregivers' health-related quality of life and productivity, ${ }^{7}$ the disease also carries an important social burden. ${ }^{8}$ In a recent burden-of-illness study conducted in five European countries (France, Germany, Italy, Spain, and the United Kingdom), the mean yearly cost per patient with MS increased with disease severity: between $€ 13,534$ and $€ 22,461$ for patients with mild MS (Expanded Disability Disease Scale [EDSS] score $\leq 3$ ); between $€ 28,524$ and $€ 43,948$ for moderate MS (EDSS 4-6.5); and between $€ 39,592$ and $€ 65,395$ for severe MS (EDSS $\leq 7$ ). ${ }^{9}$ Relapses were positively associated with increase in cost. ${ }^{7}$

There is no cure for MS at present and available diseasemodifying therapies (DMTs) aim to reduce relapses or slow down disease progression. ${ }^{10}$ In these strategies, adherence is key to obtaining optimal clinical outcomes. ${ }^{11,12}$ Poor adherence is a problematic and challenging issue in the management of many chronic diseases and is associated with significant consequences in both clinical and economic outcomes. ${ }^{11}$ Adherence to treatment in patients with chronic diseases is as low as $50 \%$, which can decrease to $40 \%$ in patients with MS. ${ }^{13}$ The main causes of nonadherence reported by patients are forgetting to take a dose, injection-site reactions (ISRs), fatigue due to the medication, dislike of the administration route, and side effects..$^{13}$

Inadequate adherence represents a significant burden, not just to patients, but also to the health care system and society. Although different studies have shown the importance of treatment adherence, persistence, and compliance in reducing relapse rates in patients with MS, ${ }^{11}$ information on their impact on MS costs is scarce. In order to assess costs related to patient adherence to - and persistence and compliance with - DMTs, a literature review was performed. Additionally, satisfaction with and preferences for DMTs and their delivery devices were assessed, as they can affect patients' adherence, persistence, and compliance.

\section{Methods}

A comprehensive review of the literature on the costs related to adherence and persistence in MS patients taking DMTs and patient-reported outcomes (PROs) related to MS treatment (satisfaction and preferences) was performed using information from electronic databases covering a period of 6 years (January 2007-January 2013). This time period was selected in order to identify the most up-to-date and recent publications and to obtain accurate insight into current treatment options for MS from the patient perspective. In recent years, new diagnostic criteria and techniques, as well as novel treatments, have compelled us to readdress and reappraise our understanding and management of MS. ${ }^{14}$ For this reason, this review focuses on publications from the last 6 years. The source of peer-reviewed publications was MEDLINE/PubMed, using the search terms summarized in Table S1. Grey literature (Google Scholar) and congress proceedings (annual congresses of the International Society of Pharmacoeconomics and Outcomes Research, the European Committee for Treatment and Research in Multiple Sclerosis, and the European Federation of Neurological Societies) were searched. The bibliographic references of the reviewed publications were also checked.

Original articles, reviews, and congress proceedings in English or Spanish, published between January 2007 and January 2013 in Europe, North America, or Australia were included in the present review if they reported cost results related to adherence persistence and compliance in MS patients, or MS patients' satisfaction with and preference for DMTs and treatment administration devices. The review excluded cost estimations and economic evaluations of concrete active treatments; studies that reported adherence measures not related to MS costs; studies related to satisfaction with or preferences for non-DMTs; and letters to the editor, editorials, expert opinion pieces, and case studies. The study selection was performed by two independent researchers (MC and SP) and discrepancies were solved by consensus.

The methodological quality of the studies was appraised by assigning a level of evidence and recommendation based on the Centre for Evidence-Based Medicine (CEBM) criteria. ${ }^{15}$ Journal impact factor (2012) and the number of citations (Web of Science ${ }^{16}$ and Google Scholar ${ }^{17}$ ) were used to assess the probability of published results reaching a sizeable audience.

For cost studies, all cost results were converted to Euros and updated to 2013 values to facilitate the comparison of findings among publications. 


\section{Results}

A total of 398 titles were initially identified, of which 311 were excluded as they were duplicates or not relevant. After application of the inclusion/exclusion criteria, 12 publications were found to contain original information on MS-related costs associated with adherence and persistence in patients on DMTs, and were therefore included in the present review (Figure 1).

Four retrospective cohort studies, one systematic review, and one decision-analytic model, all performed in the United States between 2010 and 2013, were reviewed. Moreover, in order to understand the determinants of patient satisfaction with therapeutic alternatives that may have a great impact on adherence, compliance, and persistence with therapy over time, six other studies on satisfaction with and preferences for DMTs and their devices were included. Five studies were performed in Europe (two in Switzerland and one each in Germany, France, and the United Kingdom), and one in the USA between 2007 and 2012. A description of the selected studies is summarized in Table 1. Most of the studies had a level of evidence of $2 \mathrm{C}$ or lower, with a CEBM recommendation of grade $\mathrm{B}$, which implies limited methodological consistency.

In all selected studies, patient adherence to DMTs was assessed using the medication possession ratio (MPR), calculated as the total days of medication supply dispensed divided by the number of days that the patient should have been taking the medication. ${ }^{18}$ Provided that the number of days' supply remains constant, the longer the duration of time between the first and last prescription, the lower the MPR. Patients with an MPR of $80 \%$ or higher were considered to be adherent. Medication persistence was defined as the time duration from initiation to discontinuation of therapy. ${ }^{19}$

During the development of the present study, a systematic review assessing the published data on adherence to DMTs and its impact on both clinical and economic outcomes from the patient and payer perspective was published (January 2013). ${ }^{20}$ This publication included 24 studies related to DMT adherence. The authors reported that adherence to DMTs ranges from $21 \%-88 \%$, and that there is a numerically greater risk of MS relapses or disease progression in patients who are nonadherent to treatment (statistically significant in two of four studies). In addition, two studies showed a statistically significant reduction in the use of inpatient or emergency department services and total MS-related medical costs among patients adherent to treatment compared with patients who were nonadherent. The authors concluded that, because of the chronic nature of MS, long-term adherence to DMTs can be challenging. The authors also found that adherence was higher in studies with prospective rather than

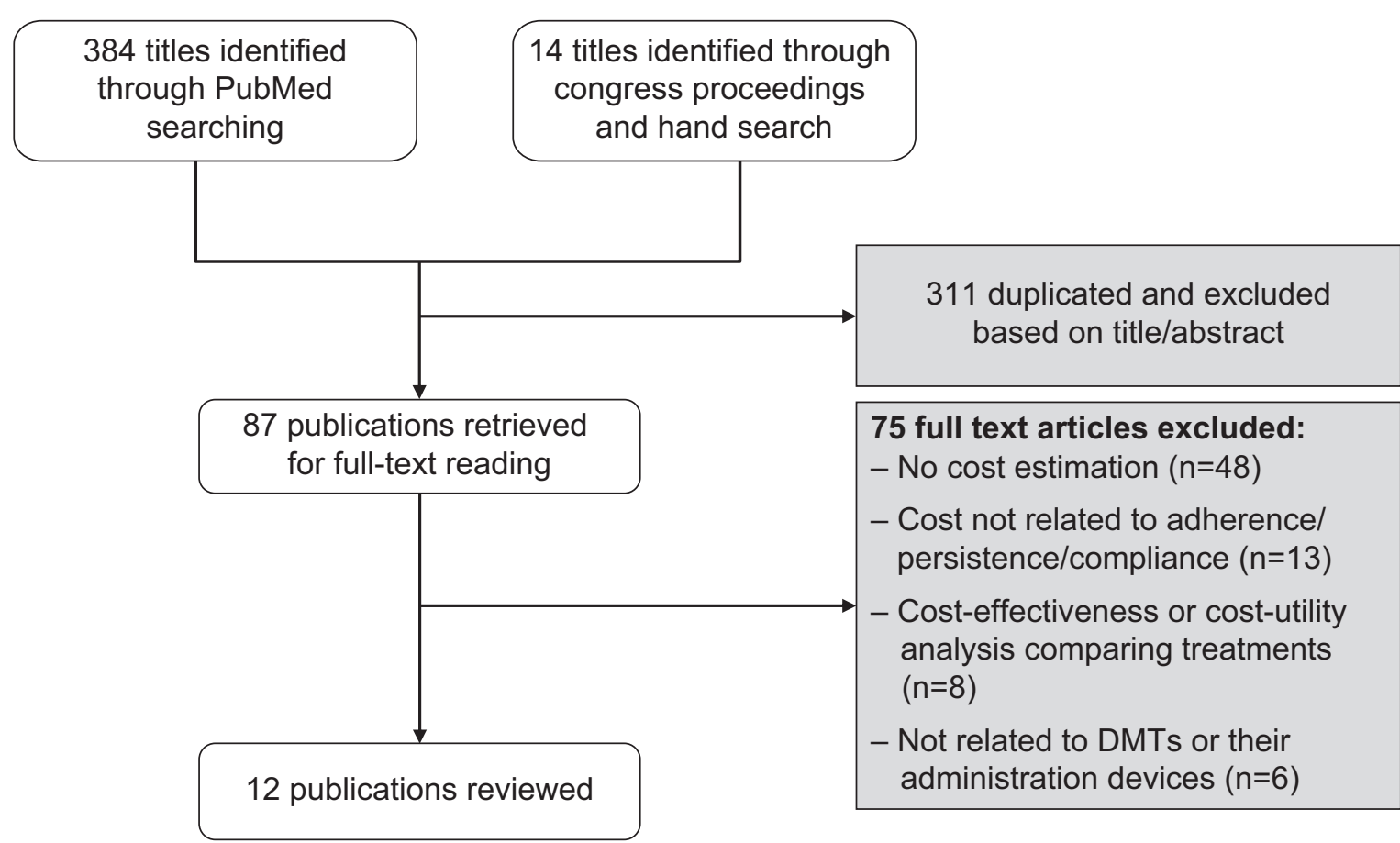

Figure I Flow-chart summary of literature search. Abbreviations: DMT, disease-modifying therapy; n, number. 


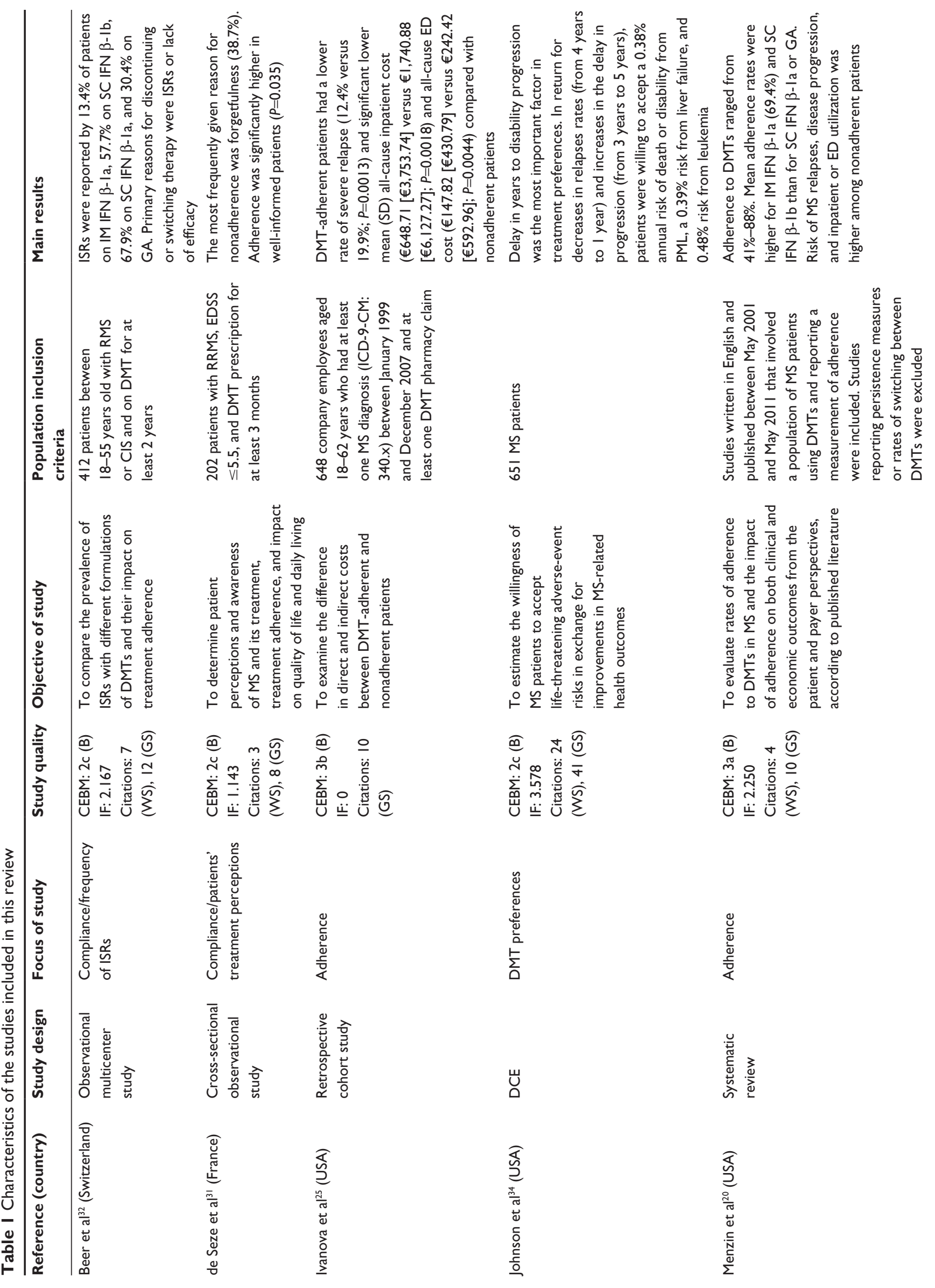



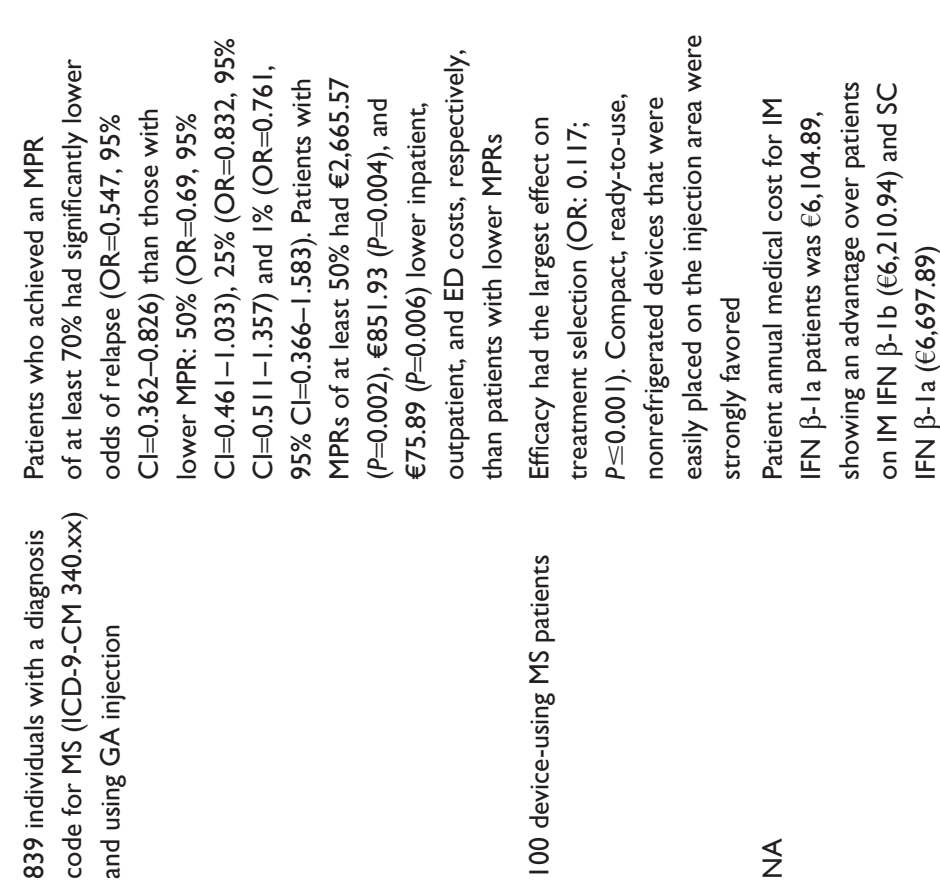

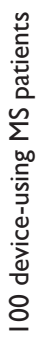

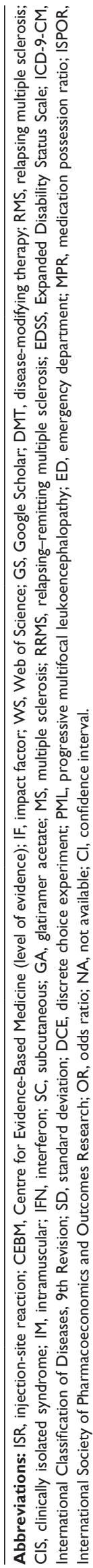

器䇰
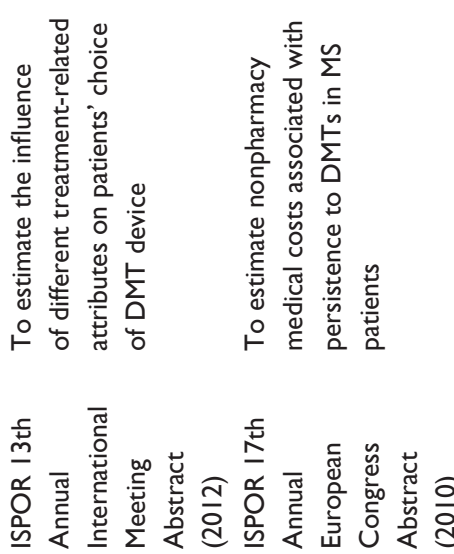

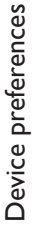

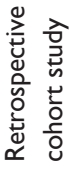
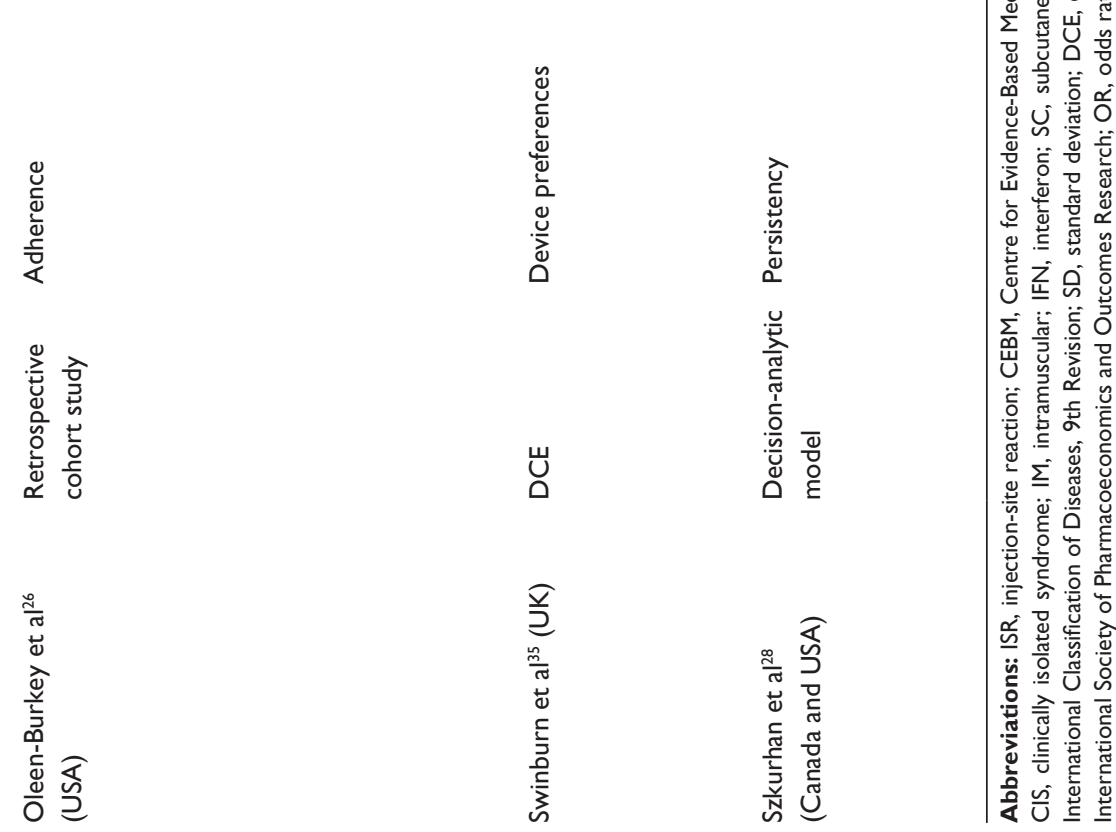

Ư

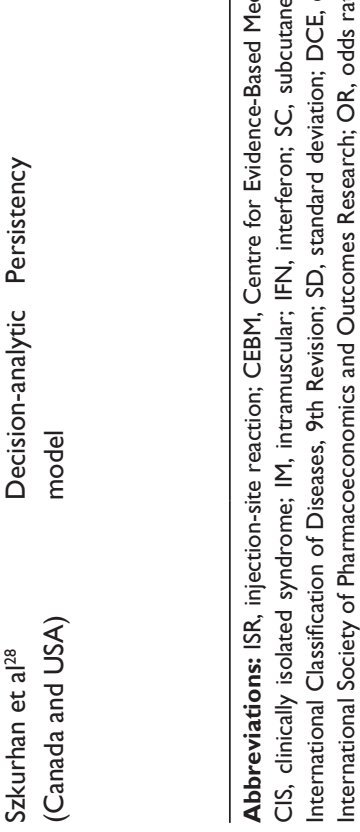


retrospective designs. Unlike the present study, this publication focused only on patients' adherence to therapy.

\section{Clinical outcomes related to adherence to and persistence with DMTs}

Relapses are the hallmark of MS, with approximately $80 \%$ of cases diagnosed as relapsing-remitting MS at onset, and relapses play an important role in determining subsequent prognosis and the development of disability. ${ }^{21}$ Some authors have reported that up to $49 \%$ of patients with relapsingremitting MS exhibit residual deficits on the EDSS after relapses, and have suggested that therapies that are effective in reducing relapse frequency and/or severity could slow or prevent worsening of the disability if initiated prior to disease onset or early in the disease course. ${ }^{22}$

DMTs have the potential to alter the natural history of MS by reducing the frequency and severity of relapses and slowing disability progression. However, patients who do not adhere to or persist with therapy are unlikely to receive the full benefits of treatment. ${ }^{23}$

Two of the studies that evaluated the association between adherence rates and MS-related costs determined the clinical outcomes related to adherence. ${ }^{24,25}$ Both studies reported that adherent patients initiating DMTs were significantly less likely to experience relapses, indicating control of symptoms or improvement in the first 12 months of follow-up (odds ratio [OR]: $0.71 ; 95 \%$ confidence interval [CI]: $0.59-0.85$; with adherent patients defined as having an MPR of $\geq 80 \%$ ). ${ }^{24}$ As Figure 2 shows, in both studies patients who were adherent to DMTs had a lower rate of severe relapses compared with nonadherent patients (27.3\% versus $34.7 \% ; P<0.001 ;^{24}$ $12.5 \%$ versus $19.5 \%$, respectively; $P=0.0200^{25}$ ). Therefore, these authors suggested that adherence to therapy could be a key factor in improving patient clinical outcomes, as observed via lower rates of MS-related symptoms in the form of relapses. ${ }^{24}$

\section{MS costs related to adherence to and persistence with DMTs}

The impact of adherence to DMTs on economic outcomes among MS patients was assessed in two retrospective cohort studies. The first study determined the impact of treatment adherence on MS-related medical resource use (inpatient, emergency department visits, MS relapses) and medical costs, ${ }^{24}$ whereas the second study compared rates of severe relapses and total direct and indirect costs over a 2-year period in US-based employees with MS who were adherent and nonadherent to DMTs. ${ }^{25}$

In both studies, the lower incidence of relapses - in particular, severe relapses - resulted in a significant decrease in health care resource use: between $35 \%{ }^{25}$ and $40 \%{ }^{24}$ in MS-related hospitalizations and between $20 \%{ }^{25}$ and $40 \%{ }^{24}$ in MS-related emergency department visits. Adherent patients were less likely to have MS-related hospitalizations (OR: 0.63; 95\% CI: 0.47-0.83) and MS-related emergency department visits (OR: 0.80; 95\% CI: 0.60-1.07) compared with nonadherent patients (Figure 2). These results suggest that adherence to therapy may be a key driver in improving patient outcomes. ${ }^{24}$

In addition to a reduction in the use of resources, medical expenditures were also lower for adherent patients when compared with nonadherent patients. Over a 2-year period, DMT-adherent patients had incurred significantly lower all-cause inpatient costs (mean [standard deviation

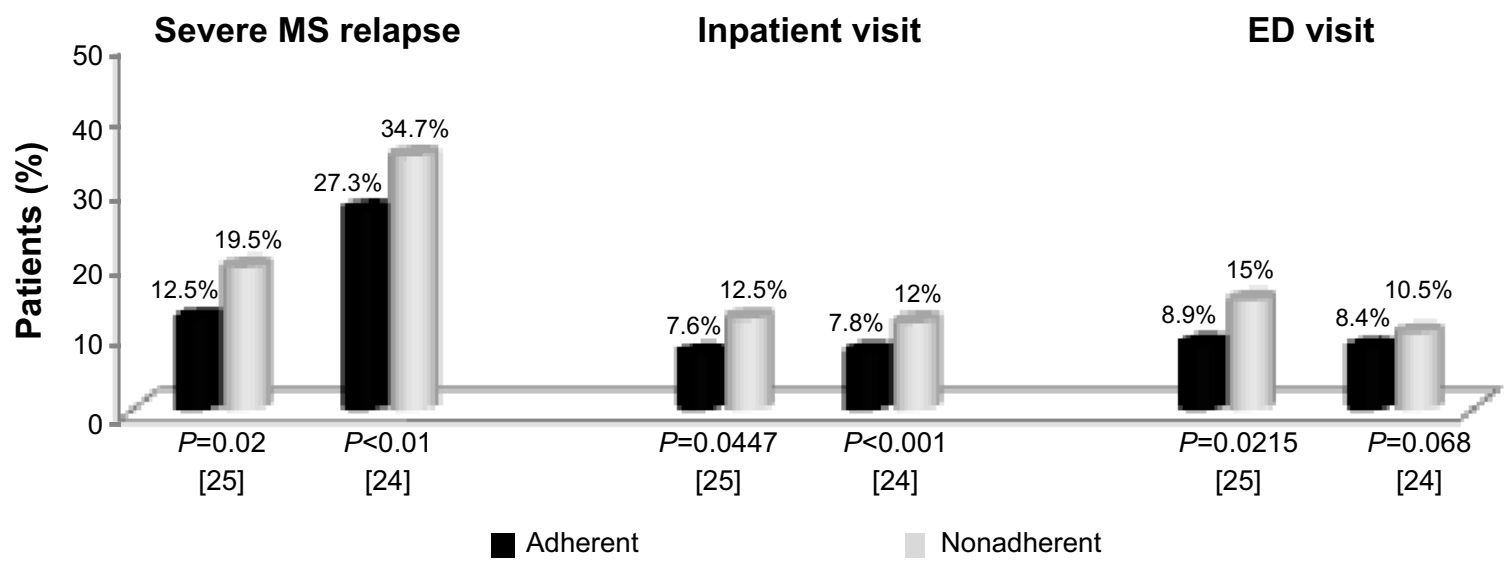

Figure 2 Comparison between the percentage of adherent and nonadherent patients with at least one severe multiple sclerosis relapse, inpatient visit, and emergency department visit over I year.

Note: Data from Tan et $\mathrm{al}^{24}$ and Ivanova et al. ${ }^{25}$

Abbreviations: MS, multiple sclerosis; ED, emergency department. 
$\{\mathrm{SD}\}]$ : $€ 648.71[€ 3,753.74]$ versus $€ 1,740.88$ [€6,127.27]; $P=0.0018])$ and all-cause emergency department costs ( $€ 147.82$ [€430.79] versus $€ 242.42$ [€592.96]; $P=0.0044]$, compared with nonadherent patients). ${ }^{25}$ When total direct costs (excluding DMT costs) were assessed, both all-cause direct costs (mean [SD]: $€ 9,337.82$ [€11,816.55] versus $€ 10,312.19[13,186.48] ; P=0.8753)$ and MS-related direct costs $(€ 4,824.58[€ 7,208.43]$ versus $€ 5,145.82$ [€8,240.16]; $P=0.4858$ ) were lower, albeit not significantly, in adherent patients compared with nonadherent patients. ${ }^{25}$ Indirect costs were also lower, but not significantly, in adherent patients compared with nonadherent patients (mean [SD]: $€ 3,012.15$ $[€ 7,745.70]$ versus $€ 3,522.44[€ 8,056.07] ; P=0.9010)$. Other authors reported that adherent groups had on average $22 \%$ (95\% CI: 8\%-34\%) less MS-related medical costs than nonadherent groups during a 12-month period $(€ 2,779.91$ [95\% CI: $2,505.12-3,084.11]$ versus $€ 3,575.92$ [95\% CI: $3,148.26-4,062.80])^{24}$

A third study reported how changes in MPR affected the probability of MS relapses and total costs and MS-related charges among patients treated with glatiramer acetate. ${ }^{26}$ Patients achieving MPRs of $50 \%$ or higher had significantly lower inpatient and emergency department expenditures compared with patients with lower MPRs ${ }^{26}$ incurring $€ 2,665.57$ ( $P=0.002), € 851.93$ ( $P=0.004)$, and $€ 75.89(P=0.006)$ lower inpatient, outpatient, and emergency department costs, respectively, than patients with MPRs of less than $50 \%$. The impact of adherence on total cost became greater as the MPR threshold increased. Patients with an MPR threshold of at least $95 \%$ reached incremental benefits of $€ 6,728.72$ ( $P \leq 0.001), € 10,297(P=0.001)$, and $€ 87.51(P=0.05)$ in inpatient, outpatient, and emergency department costs, respectively. ${ }^{26}$

The fourth study evaluated the clinical and economic impact of specialty care management programs designed to increase adherence among patients with MS. Results showed that implementation of a special care management program during 12 months, using educational materials and reminder calls, led to an increase in the adherence ratio of up to $18 \%$ (95\% CI: $16 \%-19 \%)$ and longer persistence with treatment (306.1 days versus 246.9 days; $P<0.001)$ among participants with respect to controls. ${ }^{27}$ Participants had both lower hospitalization rates $(7.1 \%$ versus $12.0 \% ; P<0.001)$ and lower hospitalization risk (OR: $0.51 ; 95 \%$ CI: 0.39-0.67) than controls. As a consequence, the nonpharmacy costs decreased by $€ 217.12$ per patient during 1-year of follow-up, but increased by $€ 1,263.25$ per patient in the control group. Nevertheless, the pharmacy cost increase due to improved adherence and persistence caused the total health care cost to increase by $21 \%$, suggesting that the global economic benefit of this program should be assessed on a longer time horizon. ${ }^{27}$

A decision-analytic model designed to determine the economic impact of DMT persistence on 2-year nonpharmacy medical costs, using persistence rates and health care resource use and costs from published literature, showed that patients initiated on intramuscular interferon (IFN) $\beta$-1a had $8.9 \%$ lower annual medical costs $(€ 6,104.89)$ than did patients initiated on subcutaneous (SC) IFN $\beta-1 b(€ 6,697.89)$ and $1.7 \%$ lower costs than those on SC IFN $\beta-1 \mathrm{a}(€ 6,210.94){ }^{28}$ These results indicated that persistence with DMTs was an important factor in overall medical costs for patients with MS. For this reason, efforts to improve persistence with DMTs could lead to lower medical costs for health care systems. ${ }^{28}$

\section{PROs related to adherence: treatment satisfaction and preferences for attributes of DMT delivery devices}

Inadequate adherence reduces the effectiveness of treatment, which can lead to relapses and deterioration in general health. Different studies have shown a positive association between treatment satisfaction and adherence, compliance, or persistence, with the most satisfied patients being the most adherent, compliant, or persistent. ${ }^{29}$ Consequently, understanding patient perceptions of and preferences for therapies may help in developing strategies to improve adherence, compliance, and persistence with treatment. Six studies were reviewed to identify the determinants of patient satisfaction that may have an impact on adherence, compliance, and persistence.

\section{Determinants of discontinuing MS treatment}

Three studies investigated the reasons for discontinuing MS treatment. The first, performed in Germany, estimated the compliance, satisfaction, and adverse effects of DMTs. ${ }^{30}$ The second, a French study, assessed patient perceptions and awareness of MS and its treatment, treatment adherence, and impact of treatment on quality of life and daily living. ${ }^{31}$ The third was a 2-year, observational, multicenter study conducted in Switzerland (the Swiss MS Skin Project) that evaluated the relative frequency and severity of ISRs associated with injectable DMTs. ${ }^{32}$

In Germany, about $75 \%$ of patients were compliant with DMTs for more than 2 years. Only $4.2 \%$ reported discontinuing treatment during the first 3 months, 9\% during the first year, and $11.3 \%$ by 2 years of treatment. During a period 
of less than 4 months, treatment was suspended once in $75.7 \%$ of patients, twice in $8.5 \%$, thrice in $2.6 \%$, and more than three times in $3.4 \%$. Further, treatment had been changed once in $75.3 \%$ of patients, twice in $10.9 \%$, thrice in $10.5 \%$, and more than three times in $3.3 \% .{ }^{30}$ Overall treatment satisfaction with DMTs among German patients with MS was high to moderate (score: $2.1-2.7$, where $1=$ highest satisfaction and $6=$ worst satisfaction). ${ }^{30}$

In both the German and French studies, patients reported that the main reasons for discontinuing DMTs were adverse effects (26.4\% and 54\%, respectively), ${ }^{30,31}$ physician's recommendation $(24.7 \%),{ }^{30}$ or a lack of treatment effect $(23.3 \%$ and $46 \%){ }^{30,31}$ However, regression analyses of factors influencing treatment compliance performed in Germany revealed that only the use of a wheelchair and the secondary progressive course of MS significantly predicted a lower compliance with treatment. ${ }^{30}$

The Swiss MS Skin Project reported that ISRs were one of the most common reasons for patients discontinuing or switching therapy $(30.8 \%$ at the first evaluation and $17.9 \%$ after 1 year). ${ }^{32}$ The authors recommended selecting therapies associated with a lower ISR risk and educating patients on strategies to minimize the occurrence of ISRs, with the aim of improving treatment adherence and thus increasing the chance of optimal MS treatment outcomes over the long term. ${ }^{32}$

To increase compliance and satisfaction with treatment, adequate information about the disease, therapeutic options, handling of medications, and side effects and their management were necessary. ${ }^{30}$ In France, less than half (42.6\%) of patients with MS reported that they had been well informed about their disease, while $34.7 \%$ reported that they had been well informed about their treatment. Nearly half of patients (44\%) were involved in the treatment decision-making process and listed efficacy (42.2\%), injection frequency (27.8\%), and adverse events $(15.6 \%)$ as the most important factors when choosing a treatment over another. ${ }^{31}$

\section{Patient satisfaction with delivery device}

MS patients' satisfaction with their current injection device and their reaction to a new autoinjector were investigated in a multicountry study performed in Canada, France, Germany, Italy, Spain, and the US. ${ }^{33}$ The results showed that less than half of patients were satisfied with their current delivery device $(40 \%$, defined as $8-10$ points on a 10 -point scale where $0=$ "not at all satisfied" and $10=$ "very satisfied"). The proportion of "very satisfied" patients was highest among those using an autoinjector device (46\%), intermediate in patients using a prefilled syringe (39\%), and lowest in patients using a syringe and vial $(23 \%)$. Of the respondents, $66 \%$ always self-injected the medication, 19\% always had someone else perform the injection, and the remaining $15 \%$ sometimes self-injected and other times had someone else do it. The reasons for not self-injecting were physical (the most common being "difficulty with injecting" [57\%] and pain at injection being infrequent $[10 \%]$ ) or psychological (including dislike of looking at needles [39\%], the thought of injection [37\%], and lack of confidence in one's ability to inject correctly [32\%]). ${ }^{33}$

When those patients were asked to consider a new autoinjection device, they listed the most positive features as: the possibility of adjusting the speed and depth of injection (39\%); a dosing log for reliable dose monitoring (38\%); easily accessible injection areas (34\%); a simple uncomplicated procedure (29\%); and a hidden needle (29\%). Overall, $96 \%$ of respondents had identified a benefit that would encourage them to ask their nurse or physician about the new device, and $23 \%$ considered that the new device had no drawbacks at all. ${ }^{33}$ These results suggest that technologies designed to help patients overcome physical and psychological barriers to self-injection may contribute to improved treatment adherence. $^{33}$

\section{Patient preferences for MS health states and for attributes of MS treatments}

Two publications referred to discrete choice experiments (DCE) that evaluated preferences for treatment attributes and reviewed health states of patients with MS. The first DCE estimated the willingness of patients to accept life-threatening adverse-event risks in exchange for improvements in their MS-related health outcomes. ${ }^{34}$ Five treatment attributes were selected: two measures of treatment efficacy (reduction in the number of relapses experienced in the next 5 years, and delay [in years] of disability progression); and three mortality risks (death or severe disability from progressive multifocal leukoencephalopathy [PML], death from liver failure, and death from leukemia). The treatment attribute that had the largest overall effect on preferences was years to progression, followed by the risk of PML, whereas the attribute with the smallest overall effect was the frequency of relapses over 5 years. Patients said that they were willing to assume associated mortality risks as high as $0.39 \%(95 \% \mathrm{CI}: 0.32 \%-0.46 \%)$ for liver failure, $0.38 \%$ (95\% CI: $0.32 \%-0.43 \%$ ) for PML, and $0.48 \%$ (95\% CI: $0.39 \%-0.58)$ for leukemia in order to obtain a relevant clinical benefit, defined as a 5-year reduction in MS relapses from 4 years to 1 year and a slowing down of 
MS progression from 3 years to 5 years. Thus, most patients with MS indicated that they were willing to accept risks in exchange for clinical efficacy.

The second DCE, performed in the UK, assessed MS patients' preferences for the attributes of injection devices..$^{35}$ Choice sets of attributes identified in the literature - including ease of use, comfort of use, additional functions, needle visibility, practicality, and efficacy (expressed in terms of prevention of increasing disability level) - were presented as pairs of hypothetical treatments. Participants were asked to decide which of the two treatments they preferred. Results determined that efficacy was the attribute with the highest impact on patient preference (OR: 0.117 for a device that was likely to result in disease progression by one level on the MS scale; $P \leq 0.001$ ). These patients placed great importance on device comfort and practicality, preferring compact, readyto-use, nonrefrigerated devices that could be easily placed on the injection site.

\section{Discussion}

This review of the literature on adherence to MS treatments and disease costs shows the scarcity of studies addressing this issue. Nonetheless, the available publications allow us to anticipate the magnitude in cost reduction that can be achieved by slight improvements in treatment adherence.

Nonadherence to medication is a significant problem in chronic disease management, ${ }^{36}$ and patients who do not adhere to or persist with therapy are unlikely to receive the full benefits of treatment, leading to worse clinical outcomes. ${ }^{23}$ As this review shows, patients who are adherent to DMTs are less likely to experience MS relapses than those who are nonadherent. Regardless of its effects on long-term disability, preventing MS relapses has positive effects on patients' short-term quality of life and functioning. ${ }^{9}$ When relapses occur, treatment choice depends on relapse severity and may include additional visits to outpatient services and inpatient care. ${ }^{37}$ Lage et a ${ }^{38}$ observed that severe MS relapses requiring hospitalization were associated with high medical costs, and that nonadherence to DMTs was strongly associated with an increased number of severe relapses.

Although treatment characteristics are key to adherence among patients with MS, adherence is a much more complex phenomenon. Mood or anxiety disorders increased by almost five times the likelihood of exhibiting adherence problems with DMTs compared with the absence of a psychiatric diagnosis. ${ }^{35}$ Poor adherence has also been associated with memory difficulties, anxiety, depression, neuroticism, and low conscientiousness. ${ }^{39}$ The design of the reviewed studies also influenced adherence and cost results. Retrospective studies have been reported to draw less satisfactory adherence estimates than prospective studies. ${ }^{20}$ Most studies reviewed had a retrospective design, suggesting that there may be greater differences in costs attributable to adherence among patients with MS.

Preventing patients from having severe relapses by improving adherence to treatment also suggests fewer hospital admissions and emergency department visits, which are the most expensive medical resources. As a consequence, the nonpharmacological costs of the disease decrease significantly. Findings of this nature are common with other chronic, disabling, progressive diseases that require sustained, long-term treatments, for which injectable formulations become paramount. In this sense, a study that assessed the impact of adherence in patients with rheumatoid arthritis suggested that even though DMTs increased pharmacy costs, this cost increase was partially offset by a decrease in other sanitary costs (related to exacerbations and hospitalizations) and by improvements in health-related quality of life and treatment satisfaction. ${ }^{40}$

The positive correlation between nonadherence, adverse outcomes, and medical costs described in this review has also been documented for other chronic diseases. A retrospective analysis of women with osteoporosis indicated that low adherence (MPR $<50 \%$ ) was associated with a $37 \%$ higher likelihood of fracture, with $12 \%-18 \%$ higher all-cause medical costs and 34\%-59\% more all-cause hospitalizations. ${ }^{41}$ A study that examined the relationship between medication adherence and the use and cost of health services in patients with four chronic vascular conditions (congestive heart failure, hypertension, diabetes, and dyslipidemia) found that although adherent patients incurred higher pharmacy spending than those who were nonadherent, annual medical spending was significantly lower for adherent patients. ${ }^{42} \mathrm{As}$ anticipated, improvements in medication adherence increase pharmacy spending. Nevertheless, the additional pharmacy spending incurred from adherence was more than offset by the medical savings gained as a result of reductions in hospitalization and emergency department use. ${ }^{42}$

It is known that the introduction of DMTs increases pharmacological spending. Nonetheless, these therapeutic options are associated with improvements in quality of life and better clinical outcomes that can be translated into long-term savings. A German study that compared the cost composition of rheumatoid arthritis before and after the introduction of biological drugs showed that although pharmaceutical costs increased from $€ 550$ to $€ 1,580$ ( $P<0.001)$, overall costs 
before and after the introduction of tumor necrosis factor blockers were comparable (€4,280 to $€ 3,830 ; P=0.3)$. ${ }^{43}$ The decrease in hospitalization and productivity costs associated with better clinical outcomes is promising in terms of future long-term cost savings, suggesting that initial increased pharmacological costs may be offset over the long term.

Preferences for treatments have been shown to affect treatment satisfaction, ${ }^{44}$ and improvement in treatment satisfaction has been associated with greater treatment adherence. ${ }^{29}$ Some authors have suggested that improving the convenience and acceptability of a treatment by using best-suited drugdelivery devices is another approach to improve adherence to MS treatment. ${ }^{45}$ Ascertaining the attributes that patients most value would improve treatments and devices in a way that could increase patient adherence and satisfaction. According to the reviewed publications, the attributes that are reported as more preferable to patients when considering DMTs are treatment efficacy, administration frequency, and ISRs. Similar to other findings on the preferences for device characteristics, patients tended to assign greater importance to minor efficacy gains while gaining benefits on the administration of treatments. ${ }^{46}$ In line with these results, a recent study suggests that the provision of information at the outset of therapy may improve adherence in patients with MS, with high-quality information and well-being on treatment being the main determinants of persistence with SC IFN $\beta-1 a .{ }^{47}$

In concordance with other publications, most patients with MS indicated that they would be willing to accept risks in exchange for clinical efficacy. Patients with cancer were more willing to undergo intensive therapy with a small likelihood of benefit than were physicians or the general public, suggesting that changes in patients' health status may affect their treatment preferences as they become more willing to tolerate a diminished state of health. ${ }^{48}$ Fried et $\mathrm{al}^{49}$ found that patients who experienced a decline in instrumental activities of daily living were more likely to rate more severe functional disability as an acceptable outcome of therapy than those who did not experience such a decline.

The results of this review have to be interpreted in the context of its limitations - namely, the small number of papers identified. Despite our comprehensive search, there may have been relevant papers in languages other than English or Spanish, or that may have been indexed in databases other than PubMed, which we did not identify. The weak consistency of the methodology in most of the reviewed studies can be explained by their observational and exploratory design, which tends to translate into lower rates of methodological quality when an assessment tool, such as the CEBM level of evidence, is applied. Most reviewed studies involved North American or European populations, thus reflecting disease characteristics and viewpoints of patients in developed countries with presumably similar socioeconomic levels. A broader scope encompassing PROs and MS costs in developing countries may need to be considered to describe the disease worldwide. MS is a costly disease and the health care system differences in developing countries may distinctively hamper patient adherence to and persistence with treatment.

Although these results should not be generalized and are not necessarily applicable across different countries and within diverse health care scenarios, the review offers a glimpse into the importance of adequate adherence in MS patients to manage the costs associated with the disease and highlights the potential benefits provided by new technologies, designed to support patient engagement by providing easily accessible information to aid disease management decisions, allowing patients to record and share their experience about important physical and psychological/ emotional aspects of their disease, as well as optimizing the clinic time that patients and physicians have together. Further insight into MS management options, their economic impact, and their value from the patient perspective is needed across cultures.

\section{Disclosure}

SP, MC, LL, and JLP worked on this study that was funded by Merck, S.L. (an affiliate of Merck KGaA, Darmstadt, Germany), but they have no other conflicts of interest to disclose. DMM is an employee of EMD Serono Inc. (a subsidiary of Merck KGaA, Darmstadt, Germany) and CP is an employee of Merck, S.L. (an affiliate of Merck KGaA, Darmstadt, Germany). The authors report no other conflicts of interest in this work.

\section{References}

1. Trapp BD, Nave KA. Multiple sclerosis: an immune or neurodegenerative disorder? Annu Rev Neurosci. 2008;31:247-269.

2. Confavreux C, Vukusic S. Natural history of multiple sclerosis: a unifying concept. Brain. 2006;129(Pt 3):606-616.

3. Tullman MJ. Overview of the epidemiology, diagnosis, and disease progression associated with multiple sclerosis. Am J Manag Care. 2013;19 (2 Suppl):S15-S20.

4. Milo R, Kahana E. Multiple sclerosis: geoepidemiology, genetics and the environment. Autoimmun Rev. 2010;9(5):A387-A394.

5. Kingwell E, Marriott JJ, Jetté N, et al. Incidence and prevalence of multiple sclerosis in Europe: a systematic review. BMC Neurol. 2013;13:128.

6. Alcalde-Cabero E, Almazán-Isla J, García-Merino A, de Sá J, de PedroCuesta J. Incidence of multiple sclerosis among European Economic Area populations, 1985-2009: the framework for monitoring. BMC Neurol. 2013;13:58. 
7. Benito-León J. Multiple sclerosis: is prevalence rising and if so why? Neuroepidemiology. 2011;3(3-4):236-237.

8. World Health Organization. Atlas: Multiple Sclerosis Resources in the World 2008. Geneva, Switzerland. World Health Organization; 2008. Available from: http://www.who.int/mental_health/neurology/ Atlas_MS_WEB.pdf. Accessed December 18, 2013.

9. Karampampa K, Gustavsson A, Miltenburger C, Eckert B. Treatment experience, burden and unmet needs (TRIBUNE) in MS study: results from five European countries. Mult Scler. 2012;18(2 Supp1):7-15.

10. Markowitz CE. The current landscape and unmet needs in multiple sclerosis. Am J Manag Care. 2010;16(8 Suppl):S211-S218.

11. Bandari DS, Sternaman D, Chan T, Prostko CR, Sapir T. Evaluating risks, costs, and benefits of new and emerging therapies to optimize outcomes in multiple sclerosis. J Manag Care Pharm. 2012;18(9):1-17.

12. Steinberg SC, Faris RJ, Chang CF, Chan A, Tankersley MA. Impact of adherence to interferons in the treatment of multiple sclerosis: a non-experimental, retrospective, cohort study. Clin Drug Investig. 2010;30(2):89-100.

13. Treadaway K, Cutter G, Salter A, et al. Factors that influence adherence with disease-modifying therapy in MS. J Neurol. 2009;256(4):568-576.

14. Zajicek J. Diagnosis and disease modifying treatments in multiple sclerosis. Postgrad Med J. 2005;81(959):556-561.

15. Centre for Evidene-Based Medicine [homepage on the Internet]. CEBM. Oxford, UK: Centre for Evidence-Based Medicine; 2014. Available from: http://www.cebm.net/?o=1025. Accessed June 5, 2014.

16. Web of Science [homepage on the Internet]. Web of Science. Philadelphia, PA: Thomson Reuters; 2014. Available from: http://wokinfo. com/. Accessed June 5, 2014.

17. Google Scholar [homepage on the Internet]. Google scholar. Mountain View, CA: Google Inc.; 2014. Available from: http://scholar.google. es/. Accessed June 5, 2014.

18. Lehmann A, Aslani P, Ahmed R, et al. Assessing medication adherence: options to consider. Int J Clin Pharm. 2014;36(1):55-69.

19. Cramer JA, Roy A, Burrell A, et al. Medication compliance and persistence: terminology and definitions. Value Health. 2008;11(1):44-47.

20. Menzin J, Caon C, Nichols C, White LA, Friedman M, Pill MW. Narrative review of the literature on adherence to disease-modifying therapies among patients with multiple sclerosis. J Manag Care Pharm. 2013;19(1 Suppl A):S24-S40.

21. Noseworthy JH, Lucchinetti C, Rodriguez M, Weinshenker BG. Multiple sclerosis. N Engl J Med. 2000;343(13):938-952.

22. Hirst C, Ingram G, Pearson O, Pickersgill T, Scolding N, Robertson N. Contribution of relapses to disability in multiple sclerosis. JNeurol. 2008; 255(2):280-287.

23. Patti F. Optimizing the benefit of multiple sclerosis therapy: the importance of treatment adherence. Patient Pref Adherence. 2010;4:1-9.

24. Tan H, Cai Q, Agarwal S, Stephenson JJ, Kamat S. Impact of adherence to disease-modifying therapies on clinical and economic outcomes among patients with multiple sclerosis. Adv Ther. 2011;28(1):51-61.

25. Ivanova JI, Bergman RE, Birnbaum HG, Phillips AL, Stewart M, Meletiche DM. Impact of medication adherence to disease-modifying drugs on severe relapse, and direct and indirect costs among employees with multiple sclerosis in the US. J Med Econ. 2012;15(3):601-609.

26. Oleen-Burkey MA, Dor A, Castelli-Haley J, Lage MJ. The relationship between alternative medication possession ratio thresholds and outcomes: evidence from the use of glatiramer acetate. J Med Econ. 2011; 14(6):739-747.

27. Tan H, Yu J, Tabby D, Devries A, Singer J. Clinical and economic impact of a specialty care management program among patients with multiple sclerosis: a cohort study. Mult Scler. 2010;16(8):956-963.

28. Szkurhan A, Malik S, Dembek C, Agarwal S, Rajagopalan K, Rashid N. Economic impact of persistence to disease modifying therapies for the treatment of Multiple Sclerosis. Value Health. 2010; 13(7):A250-A480. Abstract.

29. Barbosa CD, Balp MM, Kulich K, Germain N, Rofail D. A literature review to explore the link between treatment satisfaction and adherence, compliance, and persistence. Patient Prefer Adherence. 2012;6:39-48.
30. Twork S, Nippert I, Scherer P, Haas J, Pöhlau D, Kugler J. Immunomodulating drugs in multiple sclerosis: compliance, satisfaction and adverse effects evaluation in a German multiple sclerosis population. Curr Med Res Opin. 2007;23(6):1209-1215.

31. de Seze J, Borgel F, Brudon F. Patient perceptions of multiple sclerosis and its treatment. Patient Prefer Adherence. 2012;6:263-273.

32. Beer K, Müller M, Hew-Winzeler AM, et al. The prevalence of injection-site reactions with disease-modifying therapies and their effect on adherence in patients with multiple sclerosis: an observational study. BMC Neurol. 2011;11:144.

33. Verdun di Cantogno E, Russell S, Snow T. Understanding and meeting injection device needs in multiple sclerosis: a survey of patient attitudes and practices. Patient Prefer Adherence. 2011;5:173-180.

34. Johnson FR, Van Houtven G, Ozdemir S, et al. Multiple sclerosis patients' benefit-risk preferences: serious adverse event risks versus treatment efficacy. J Neurol. 2009;256(4):554-562.

35. Swinburn P, Shingler S, Ali S, Perard R, Lloyd A. Preferences of multiple sclerosis patients for attributes of self-injection devices. Value Health. 2012;15:A1-A276. Abstract.

36. Yeaw J, Benner JS, Walt JG, Sian S, Smith DB. Comparing adherence and persistence across 6 chronic medication classes. J Manag Care Pharm. 2009;15(9):728-740.

37. O'Brien JA, Ward AJ, Patrick AR, Caro J. Cost of managing an episode of relapse in multiple sclerosis in the United States. BMC Health Serv Res. 2003;3(1):17.

38. Lage MJ, Carroll CA, Fairman KA. Using observational analysis of multiple sclerosis relapse to design outcomes-based contracts for disease-modifying drugs: a feasibility assessment. J Med Econ. 2013;16(9):1146-1153.

39. Bruce JM, Hancock LM, Arnett P, Lynch S. Treatment adherence in multiple sclerosis: association with emotional status, personality, and cognition. J Behav Med. 2010;33(3):219-227.

40. Stockl KM, Shin JS, Lew HC, et al. Outcomes of a rheumatoid arthritis disease therapy management program focusing on medication adherence. J Manag Care Pharm. 2010;16(8):593-604.

41. Halpern R, Becker L, Igbal SU, Kazis LE, Macarios D, Badamgarav E. The association of adherence to osteoporosis therapies with fracture, all-cause medical costs, and all-cause hospitalizations: a retrospective claims analysis of female health plan enrollees with osteoporosis. J Manag Care Pharm. 2011;17(1):25-39.

42. Roebuck MC, Liberman JN, Gemmill-Toyama M, Brennan TA. Medication adherence leads to lower health care use and costs despite increased drug spending. Health Aff (Millwood). 2011;30(1):91-99.

43. Kirchhoff T, Ruof J, Mittendorf T, et al. Cost of illness in rheumatoid arthritis in Germany in 1997-1998 and 2002: cost drivers and cost savings. Rheumatology (Oxford). 2011;50(4):756-761.

44. Umar N, Schaarschmidt M, Schmieder A, Peitsch WK, Schöllgen I, Terris DD. Matching physicians' treatment recommendations to patients' treatment preferences is associated with improvement in treatment satisfaction. J Eur Acad Dermatol Venereol. 2013;27(6):763-770.

45. Lugaresi A. Addressing the need for increased adherence to multiple sclerosis therapy: can delivery technology enhance patient motivation? Expert Opin Drug Deliv. 2009;6(9):995-1002.

46. Palumbo A, De La Fuente P, Rodríguez M, et al. Willingness to pay and conjoint analysis to determine women's preferences for ovarian stimulating hormones in the treatment of infertility in Spain. Hum Reprod. 2011;26(7):1790-1798.

47. Syed M, Rog D, Parkes L, Shepherd GL. Patient expectations and experiences of multiple sclerosis interferon $\beta$-1a treatment: a longitudinal, observational study in routine UK clinical practice. Patient Prefer Adherence. 2014;8:247-255.

48. Donovan KA, Greene PG, Shuster JL, Partridge EE, Tucker DC. Treatment preferences in recurrent ovarian cancer. Gynecol Oncol. 2002; 86(2):200-211.

49. Fried TR, Byers AL, Gallo WT, et al. Prospective study of health status preferences and changes in preferences over time in older adults. Arch Intern Med. 2006;166(8):890-895. 


\section{Supplementary material}

Table SI PubMed search terms, strategies, and title identified

\begin{tabular}{|c|c|}
\hline Search terms & Title identified \\
\hline "Multiple sclerosis" AND "adherence" AND "cost" & 26 \\
\hline “Multiple sclerosis" AND “compliance" AND “cost" & 31 \\
\hline "Multiple sclerosis" AND "persistence" AND "cost" & 8 \\
\hline "Multiple sclerosis" AND "route of administration" AND "compliance" & 23 \\
\hline "Multiple sclerosis" AND "adherence" AND "device" & 13 \\
\hline "Multiple sclerosis" AND "route of administration" AND "adherence" & 48 \\
\hline "Multiple sclerosis" AND “device" AND “cost” & 18 \\
\hline "Multiple sclerosis" AND “device” AND “compliance" & 12 \\
\hline "Multiple sclerosis" AND “route of administration" AND “persistence" & 9 \\
\hline “Multiple sclerosis" AND "route of administration" AND "cost of disease" & 13 \\
\hline “Multiple sclerosis" AND "route of administration" AND “disease burden" & 11 \\
\hline "Multiple sclerosis" AND “treatment satisfaction" & 134 \\
\hline “Multiple sclerosis" AND “preferences" & 27 \\
\hline "Multiple sclerosis" AND "willingness to pay" & 11 \\
\hline
\end{tabular}

\section{Publish your work in this journal}

Patient Preference and Adherence is an international, peer-reviewed, open access journal that focuses on the growing importance of patient preference and adherence throughout the therapeutic continuum. Patient satisfaction, acceptability, quality of life, compliance, persistence and their role in developing new therapeutic modalities and compounds to optimize clinical outcomes for existing disease states are major areas of interest for the journal. This journal has been accepted for indexing on PubMed Central. The manuscript management system is completely online and includes a very quick and fair peer-review system, which is all easy to use. Visit http://www dovepress.com/testimonials.php to read real quotes from published authors.

Submit your manuscript here: http://www.dovepress.com/patient-preference-and-adherence-journal 